\title{
THE NUCLEAR INTERSTELLAR MEDIUM OF ULTRALUMINOUS INFRARED GALAXIES
}

\author{
P.P. VAN DER WERF \\ Leiden Observatory \\ P.O. Box 9513 \\ NL - 2300 RA Leiden \\ The Netherlands \\ (pvdwerf@strw.leidenuniv.nl)
}

\section{Introduction}

Ultraluminous infrared galaxies (ULIRGs) form the dominant component of the local galaxy population at the highest luminosities (e.g., Sanders \& Mirabel 1996 and references therein). They are major mergers of gas-rich galaxies and are characterized by a molecular interstellar medium (ISM) that is concentrated in the central kpc, where it forms a dynamically significant, or perhaps dominant, component (Scoville et al., 1991). Sanders et al. (1988) proposed that ULIRGs might harbor, or evolve into, dustenshrouded quasars, formed in their obscured centers.

\section{Near-infrared $\mathbf{H}_{2}$ emission}

Luminous vibrational $\mathrm{H}_{2}$ emission at $2.12 \mu \mathrm{m}$, first detected in the nearby ULIRGs Arp 220 and NGC 6240 (Rieke et al., 1985), is a general feature of objects of this type (Goldader et al., 1995). A near-infrared spectrum of NGC 6240 (Fig. 1) illustrates the dominance of the $\mathrm{H}_{2}$ lines, and the surprising weakness of $\mathrm{Br} \gamma$, which is also a common feature among ULIRGs. As shown by Van der Werf et al. (1993), the $\mathrm{H}_{2}$ emission in NGC 6240 peaks between the two remnant nuclei of the merging systems, and is excited by slow shocks in the dense central molecular medium. Recently, we imaged the $\mathrm{H}_{2} v=1 \rightarrow 0 \mathrm{~S}(1)$ emission in the nearby prototypical ULIRG Arp 220 (Van der Werf \& Israel 1998, see Fig. 2) and here likewise the $\mathrm{H}_{2}$ emission peaks between the two radio/near-infrared nuclei. Recent high resolution CO $J=2 \rightarrow 1$ data confirm the location of the molecular gas between the two nuclei in these galaxies, as shown by Bryant (1996) and Scoville et al. 


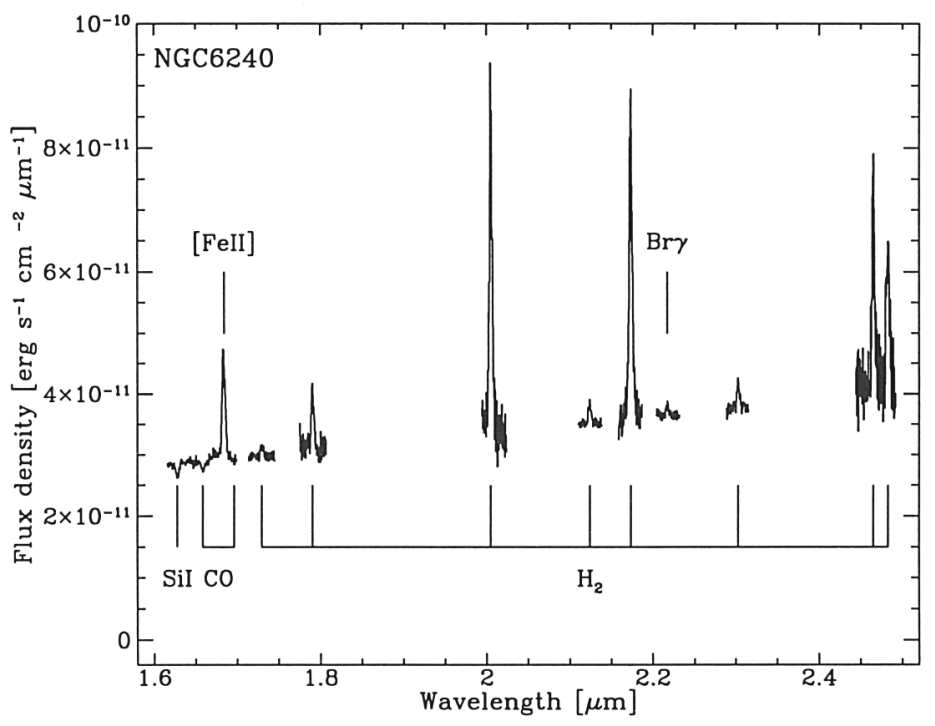

Figure 1. Spectra of the ULIRG NGC 6240 in the H- and K-bands, obtained with IRSPEC on the ESO New Technology Telescope, illustrating the presence of numerous luminous $\mathrm{H}_{2}$ vibrational lines and the faintness of $\mathrm{Br} \gamma$.

(1997, hereafter SYB97). The latter authors argue that the gas in Arp 220 is concentrated in a thin disk, where dissipation gives rise to an inward mass flux of $100-200 M_{\odot} \mathrm{yr}^{-1}$.

This dissipation is traced directly by the near-infrared $\mathrm{H}_{2}$ line emission, since the energy is dissipated by the shocks traced by this emission line. The total energy dissipated by the shocks must equal the total energy radiated away, which can be estimated from the observed $\mathrm{H}_{2} v=1 \rightarrow 0 \mathrm{~S}(1)$ emission line flux. This argument can be used to estimate an inward mass flux directly from the $\mathrm{H}_{2} v=1 \rightarrow 0 \mathrm{~S}(1)$ emission. The resulting value of $\sim 50 M_{\odot} \mathrm{yr}^{-1}$ is in reasonable agreement with the value derived from $\mathrm{CO}$, given the simplicity of the analysis.

It is tempting to speculate on a possible connection between this large inward mass flux to a position between the stellar nuclei, and the possible formation of a quasar nucleus at this location. On the other hand, the inflowing molecular gas may be consumed entirely by star formation, since the stellar nuclei, although not at the center of the molecular component, are still located within the molecular medium. The star formation rate implied by the far-infrared luminosity of Arp 220 (if entirely due to star formation) is $\sim 100 M_{\odot} \mathrm{yr}^{-1}$, in remarkable agreement with the estimates of the inward gas mass flux. Higher spatial resolution observations will be able to solve this important issue. 


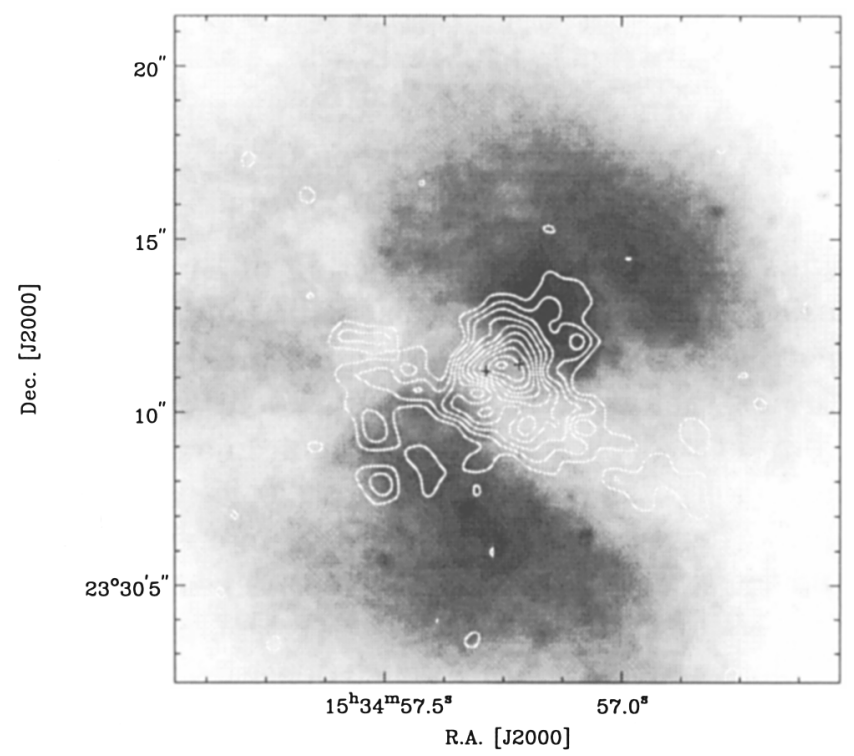

Figure 2. $\mathrm{H}_{2} v=1 \rightarrow 0 \mathrm{~S}(1)$ emission of Arp 220, shown in contours, overlaid on on F555W HST WFPC1 image. The two crosses denote the positions of the radio/near-infrared nuclei (Van der Werf \& Israel, in preparation).

\section{Nuclear star formation}

The low hydrogen recombination line luminosities of ULIRGs has been used to argue against star formation as the main power source. The situation for Arp 220 has been analyzed in some detail by SYB97, who show that the observed $L_{\mathrm{Br} \gamma} / L_{\mathrm{FIR}}$ ratio implies that only stars with masses from 5 to $25 M_{\odot}$ form, if star formation is to provide the total far-IR luminosity. In order to avoid this highly unusual situation, it is proposed that most of the bolometric luminosity of Arp 220 and other ULIRGs is produced by an obscured active nucleus. Alternatively, it has been proposed that $\operatorname{Br} \gamma$ suffers high extinction. Sturm et al. (1996) argue for an extinction $A_{\mathrm{V}} \sim 50^{\mathrm{m}}$ in front of the two nuclei, resulting in a suppression of $\mathrm{Br} \gamma$ by two orders of magnitude. However, this reasoning fails on several grounds.

1. The nuclei are visible in the near-infrared, at wavelengths as short as $1.3 \mu \mathrm{m}$ (e.g., Mazzarella et al. 1992), so that the extinction at this wavelength is at most a few magnitudes; the near-IR colours indicate a visual extinction of at most $A_{\mathrm{V}} \sim 6^{\mathrm{m}}$.

2. Adopting the high extinction proposed by Sturm et al. (1996) leads to extinction-corrected fluxes for $\mathrm{Br} \gamma$, [Ne II] $12.8 \mu \mathrm{m}$ and [S III] $33 \mu \mathrm{m}$ 
fluxes which can all be used to estimate a star formation rate (SFR). However, the resulting values increase as the wavelength of the tracer used becomes shorter. This behavior clearly indicates that the extinction estimated by Sturm et al. (1996) is too high. On the other hand, an extinction correction of only a factor of 2 on $\mathrm{Br} \gamma$ would yield consistent SFRs from $\mathrm{Br} \gamma$, [Ne II] $12.8 \mu \mathrm{m}$ and [S III] $33 \mu \mathrm{m}$.

These arguments indicate that the extinction of $\mathrm{Br} \gamma$ is moderate, and that $\mathrm{Br} \gamma$ is intrinsically faint. However, this result cannot be used to argue against star formation as the main power source in Arp 220 (as was done by SYB97), because of the importance of Lyman continuum absorption by dust within the ionized regions. The average molecular gas density in the $\sim 10^{10} M_{\odot}$ nuclear molecular complex in Arp 220 is $n_{\mathrm{H}_{2}} \sim 2 \cdot 10^{4} \mathrm{~cm}^{-3}$ (SYB97). The strong emission from high dipole moment molecules such as $\mathrm{CS}, \mathrm{HCO}^{+}$and $\mathrm{HCN}$ argues for even higher densities: $\sim 10^{10} M_{\odot}$ of molecular gas (i.e., all of the gas in the nuclear complex) has a density $n_{\mathrm{H}_{2}} \sim 10^{5} \mathrm{~cm}^{-3}$ (Solomon et al. 1990). At such densities the ionized nebulae created by hot stars are compact or ultracompact $\mathrm{H}$ II regions. However, in Galactic ultracompact $\mathrm{H}$ II regions 50 to $99 \%$ of the Lyman continuum is absorbed by dust (Wood \& Churchwell 1989). As a result, all of the usual tracers of $\mathrm{H}$ II regions (recombination lines, fine-structure lines, free-free emission) will be quenched, not extincted. Therefore, given what is known about the ISM of Arp 220 and other ULIRGs, the intrinsic faintness of $\mathrm{Br} \gamma$ and other tracers of photoionized gas is entirely expected.

\section{References}

Bryant, P.M., 1996, Ph.D. Thesis, Caltech

Goldader, J.D., Joseph, R.D., Doyon, R., \& Sanders, D.B., 1995, ApJ, 444, 97

Mazzarella, J.M., Soifer, B.T., Graham, J.R., Hafer, G., Neugebauer, G., \& Matthews, K., 1992, AJ, 103, 413

Rieke, G.H., Cutri, R.M., Black, J.H., Kailey, W.F., McAlary, C.W., Lebofski, M.J., \& Elston, R., 1985, ApJ, 290, 116

Sanders, D.B., Soifer, B.T., Elias, J.H., Madore, B.F., Matthews, K., Neugebauer, G., \& Scoville, N.Z., 1988, ApJ, 325, 74

Sanders, D.B. \& Mirabel, I.F., 1996, ARAA, 34, 749

Scoville, N.Z., Sargent, A.I., Sanders, D.B., \& Soifer, B.T., 1991, ApJ, 366, L5

Scoville, N.Z., Yun, M.S., \& Bryant, P.M., 1997, ApJ, 484, 702 (SYB97)

Sturm, E. et al., 1996, AA, 315, L133

Shaya, E.J., Dowling, D.M., Currie, D.G., Faber, S.M., \& Groth, E.J., 1994, AJ, 107, 1675

Solomon, P.M., Radford, S.J.E., \& Downes, D., 1990, ApJ, 348, L53

Van der Werf, P.P., Genzel, R., Krabbe, A., Blietz, M., Lutz, D., Drapatz, S., Ward, M.J., \& Forbes, D.A., 1993, ApJ, 405, 522

Van der Werf, P.P., \& Israel, F.P., 1998, in preparation

Wood, D.O.S., \& Churchwell, E., 1989, ApJS, 69, 831 